Nota técnica

Volumen 32(1):236-248. Enero-abril, 2021

e-ISSN 2215-3608, doi:10.15517/am.v32i1.39701

http://www.revistas.ucr.ac.cr/index.php/agromeso

\title{
Suplementación de vacas lecheras en producción con un complejo de vitaminas $B$ recubierto ${ }^{1}$
}

\section{Supplementation of dairy cows in production with a coated vitamin B complex}

\author{
Keren Gabriela Beita-Carvajal ${ }^{2}$, Jorge Alberto Elizondo-Salazar ${ }^{3}$
}

1 Recepción: 26 de noviembre, 2019. Aceptación: 22 de julio, 2020. Este trabajo formó parte de proyecto 737-B5-188, inscrito en Vicerrectoría de Investigación de la Universidad de Costa Rica.

2 Universidad Nacional de Costa Rica, Escuela de Medicina Veterinaria, Heredia, Costa Rica. gbeitac@gmail.com (https://orcid.org/00000001-6791-3320).

3 Universidad de Costa Rica, Facultad de Ciencias Agroalimentarias, Estación Experimental Alfredo Volio Mata. jorge.elizondosalazar@ucr. ac.cr (https://orcid.org/0000-0003-2603-9635).

\section{Resumen}

Introducción. Los requerimientos de vitaminas B en rumiantes son satisfechos por su propia capacidad de síntesis a nivel ruminal; sin embargo, el mejoramiento genético, los avances en nutrición animal y los altos niveles de producción actuales, puede hacer que los animales no estén llenando sus requerimientos. Objetivo. Evaluar el efecto que tiene el uso de un complejo de vitaminas del grupo B sobre la producción láctea y el metabolismo de vacas lecheras en pastoreo. Materiales y métodos. Durante el segundo semestre del 2017, se utilizaron treinta vacas de raza Jersey divididas en dos grupos que se mantuvieron durante dos periodos experimentales de 30 días. Durante el primer periodo de un mes, los animales del primer grupo recibieron 3,0 g diarios de un complejo de vitaminas B, mientras que los animales del otro grupo recibieron un placebo y para el periodo 2 se intercambiaron los tratamientos. Se midió la producción y composición láctea durante todo el periodo. Se midieron variables químicas como glucosa, betahidroxibutirato, aspartato amino transferasa, nitrógeno ureico y albúmina en sangre. Resultados. No se presentaron diferencias significativas $(\mathrm{p}>0,05)$ con respecto a la producción láctea. En el periodo 1, el grupo suplementado produjo 15,8 1 por día; mientras que el grupo control produjo 16,1 1. Las concentraciones de grasa, proteína y lactosa en el primer periodo fueron de 5,0, 3,5 y 4,4 \%, respectivamente. En el periodo 2, la producción láctea fue de 14,1 y 13,7 1 para el grupo suplementado y no suplementado, respectivamente. En ningún periodo se observaron diferencias significativas $(\mathrm{p}>0,05)$ para los parámetros de química sanguínea analizados. La concentración de glucosa fue superior a $40 \mathrm{mg} \mathrm{dl}^{-1}$ y la concentración de betahidroxibutirato fue superior a 2,0 $\mathrm{mmol} \mathrm{1}^{-1}$. Conclusión. Suplementar vacas Jersey con un complejo de vitaminas B de sobrepaso no mostró ventaja alguna con respecto a las variables productivas evaluadas.

Palabras clave: ganado de leche, nutrición de rumiantes, composición láctea, glucosa, pastoreo.

\footnotetext{
Abstract

Introduction. The requirements of B vitamins in ruminants are satisfied by their own ability to synthesize them at ruminal level. However, genetic improvement, advances in animal nutrition, and current high levels of production 
can make animals not to meet their requirements. Objective. To evaluate the effect that the use of a vitamin B complex has on dairy production and the metabolism of grazing dairy cows. Materials and methods. During the second semester of 2017, thirty Jersey cows were divided into two groups that were maintained during two 30-day experimental periods. During the first period of one month, cows in the first group received $3.0 \mathrm{~g}$ daily of a vitamin B complex, while the cows in the other group received a placebo and for period 2 the treatments were exchanged. Dairy production and composition were measured during the whole experiment. Chemical variables such as glucose, betahydroxybutyrate, aspartate aminotransferase, urea nitrogen, and blood albumin were also measured. Results. There were no significant differences $(\mathrm{p}>0.05)$ with respect to milk production. In period 1 , the supplemented group produced 15.81 of milk per day; while the control group produced 16.11 . Fat, protein, and lactose concentrations in the first period were 5.0, 3.5, and $4.4 \%$, respectively. In period 2, milk production was 14.1 and 13.71 for the supplemented and non-supplemented group, respectively. No significant differences $(p>0.05)$ were found in any of the periods for any of the analyzed parameters in blood chemistry. Glucose concentration was greater than $40 \mathrm{mg} \mathrm{dl}^{-1}$ and betahydroxybutyrate concentration was greater than $2.0 \mathrm{mmol} \mathrm{l}^{-1}$. Conclusion. Supplementing Jersey cows with a rumen protected vitamin $\mathrm{B}$ complex showed no advantage with respect to the evaluated productive variables.

Keywords: dairy cattle, ruminant nutrition, milk composition, glucose, grazing.

\section{Introducción}

Las vitaminas son un grupo de compuestos orgánicos presentes en cantidades mínimas en los alimentos, son esenciales para el correcto funcionamiento del metabolismo y su déficit en la dieta provoca una deficiencia (McDowell, 2000). Esta deficiencia causa una enfermedad específica, la cual se cura o previene cuando se suplementa la vitamina específica necesaria (Bender, 2015). Las vitaminas se clasifican en dos grupos: las liposolubles y las hidrosolubles (Baron, 2016). Las vitaminas liposolubles (vitaminas A, D, E y K) se disuelven en grasas y aceites y su exceso se almacena en el tejido graso del cuerpo, por lo que su consumo por arriba de los requerimientos puede causar toxicidad. Las vitaminas hidrosolubles (vitaminas del complejo B y vitamina C) se disuelven en agua y cuando se consumen en exceso se excretan a través de la orina. Estas vitaminas se obtienen diariamente de la dieta y no se almacenan en el cuerpo. Las vitaminas tiamina (B1), riboflavina (B2), niacina (B3), ácido pantoténico (B5), piridoxina (B6), biotina (B8), ácido fólico (B9) y cianocobalamina (B12) están relacionadas con el metabolismo celular (Richmonds, 2004).

La producción de las vacas en la actualidad es mucho más elevada que hace cincuenta años y desde entonces se han venido dando incrementos en los niveles de producción láctea y sus componentes (Santschi et al., 2005; Juchem et al., 2012). Por ejemplo, la producción por vaca en los Estados Unidos ha aumentado a más del doble en los últimos cuarenta años y en la actualidad existen vacas que producen más de $20000 \mathrm{~kg}$ de leche por lactancia (Oltenacu \& Broom, 2010). La tendencia al aumento en la producción de leche se ha venido dando como resultado de la selección genética de los animales y prácticas como la inseminación artificial (Cassell, 2001).

Es muy probable que el aumento en la producción láctea haya incrementado los requerimientos de vitaminas del complejo B de los animales y por lo tanto, la síntesis de estas vitaminas a nivel ruminal, puede no ser suficiente para llenar los requerimientos y sea necesaria su suplementación en las dietas de las vacas lecheras.

Diversas investigaciones reportan efectos benéficos con la suplementación de vitaminas del complejo B (Santschi et al., 2005), dentro de los efectos se mencionan una mejora en la producción láctea, en la composición láctea y en la salud de las pezuñas, los cuales serían de gran beneficio para los productores y que al final se podría traducir en un beneficio económico para el sector lechero del país. 
En un estudio se compararon varios tratamientos: suplementación con $0,87 \mathrm{mg}$ de biotina $\mathrm{kg}^{-1} \mathrm{de} \mathrm{MS}$, con 21 mg de ácido pantoténico por kg de MS o suplementar las dos vitaminas juntas (con las mismas dosis anteriormente mencionadas) y el grupo control; como resultado no se encontró diferencias significativas $(\mathrm{p}>0,05)$ en producción de leche entre los tratamientos aplicados (Ferreira et al., 2015).

Sin embargo, en otro estudio al suplementar un complejo de vitaminas B protegido aumentó la producción láctea $\left(0,86 \mathrm{~kg} \mathrm{día}^{-1}\right)$ con respecto al grupo control en vacas en etapa de lactación temprana (Sacadura et al., 2008). Al respecto se indicó que los animales en lactación temprana pueden responder más a la suplementación con vitaminas B, ya que en esta etapa el consumo de materia seca es menor si se compara con la producción láctea, asimismo hay menor síntesis y escape de vitaminas B del rumen (Sacadura et al., 2008). Esta última particularidad hace que pueda haber un déficit de síntesis de vitaminas B en el rumen y por ende, animales en esta etapa productiva se pueden beneficiar mejor de la suplementación.

El aumento en la producción láctea también se ha visto acompañado por una disminución de la fertilidad, un aumento en patologías podales y metabólicas, y una disminución en la longevidad del animal (Oltenacu \& Broom, 2010), si se considera que las vitaminas intervienen activamente en el metabolismo, tienen diversas funciones e impactan la salud y productividad, su uso sería de beneficio para contrarrestar estas afecciones (Sacadura et al., 2008).

El hígado es el mayor sitio de síntesis de glucosa en el rumiante y sus precursores son propionato, amino ácidos gluconeogénicos, lactato, glicerol e isómeros de butirato y valerato (Bergman et al., 1974; Leng, 1970). En condiciones de malnutrición, la contribución del propionato y otros precursores derivados de la dieta disminuye, mientras que la contribución del lactato, glicerol y amino ácidos provenientes de los tejidos aumenta, con la particularidad de que esta glucosa se produce a una tasa se síntesis más baja (Lomax \& Baird, 1983). Se ha visto que concentraciones elevadas de AGNE y BHBA en conjunto con concentraciones disminuidas de glucosa e insulina son indicativos de un balance energético negativo (van-Knegsel et al., 2005).

El objetivo de este experimento fue evaluar el efecto que tiene el uso de un complejo de vitaminas del grupo B sobre la producción láctea y el metabolismo de vacas lecheras en pastoreo.

\section{Materiales y métodos}

\section{Localización y animales}

El experimento se llevó a cabo durante el segundo semestre del 2017 en la Estación Experimental de Ganado Lechero Alfredo Volio Mata de la Universidad de Costa Rica; ubicada en el pueblo de Ochomogo perteneciente al distrito San Nicolás del cantón de la Unión, de la provincia de Cartago. Su altitud es de 1542 m, con una precipitación media anual de $2050 \mathrm{~mm}$, distribuida de mayo a noviembre y una humedad relativa media de $84 \%$. La temperatura media anual es de $19,3{ }^{\circ} \mathrm{C}$ con una máxima de $23{ }^{\circ} \mathrm{C}$ y una mínima de $13{ }^{\circ} \mathrm{C}$ (Elizondo \& Boschini, 2001).

Se contó con un hato experimental de treinta vacas en producción de la raza Jersey, que se separaron en dos grupos con base en el número de días en lactancia ( $150 \pm 50$ días), el número de partos $(3,0 \pm 2)$ y la producción diaria de leche $(16,0 \pm 2,0)$.

\section{Dieta}

Los animales se encontraban bajo un sistema de semi-pastoreo con reforzamiento de una ración parcial mezclada (RPM). La base de la alimentación era el forraje de pastoreo (pasto estrella: Cynodon nlemfuensis). Se 
utilizó el método de reversa ${ }^{4}$ para estimar el consumo de forraje por parte de los animales en el potrero y su aporte energético (Baker, 1982; National Research Council [NRC], 2001). La composición nutricional de la RPM fue: 91 $\%$ de ensilado de maíz; 2,5 \% de alimento balanceado; $2,5 \%$ de pulpa cítrica deshidratada; 2,5\% de harina de soya y $1,5 \%$ melaza de caña. Los ingredientes se homogenizaban durante 15 min en una mezcladora de capacidad de $4000 \mathrm{~kg}$ y luego se les suministraba $10 \mathrm{~kg}$ a los animales antes de cada ordeño (am y pm), para un total de $20 \mathrm{~kg}$; adicionalmente, durante cada ordeño se les ofreció $2,0 \mathrm{~kg}$ de alimento balanceado, para un total de 4,0 $\mathrm{kg}$ diarios.

Semanalmente se recolectaron muestras de la RPM que se trasladaron al laboratorio de la Estación Experimental Alfredo Volio Mata para su respectivo análisis. Los análisis efectuados fueron materia seca (MS) (humedad por estufa a $105^{\circ} \mathrm{C}$ ), proteína cruda (PC) (AOAC 2001.11), fibra en detergente ácido (FDA) (AOAC 973.18), fibra en detergente neutro (FDN) (AOAC 2002.04), lignina, celulosa, hemicelulosa y cenizas (AOAC 942.05). Los datos de la composición nutricional de los ingredientes utilizados en las dietas se muestran el Cuadro 1. Los valores nutricionales para el alimento balanceado, pulpa cítrica deshidratada y harina de soya se tomaron de la etiqueta del fabricante. La estimación de la $\mathrm{EN}_{\mathrm{L}}\left(\mathrm{Mcal} \mathrm{kg}^{-1}\right)$ se realizó mediante el modelo del NRC (2001).

Cuadro 1. Composición nutricional de los ingredientes utilizados durante el ensayo sobre el efecto que tiene el uso de un complejo de vitaminas B sobre la producción láctea y el metabolismo de vacas lecheras raza Jersey, en pastoreo. Cartago, Costa Rica. 2017.

Table 1. Nutritional composition of the ingredients used during the test on the effect that the use of a vitamin B complex has on milk production and the metabolism of grazing Jersey dairy cattle. Cartago, Costa Rica. 2017.

\begin{tabular}{lcccccc}
\hline & \multicolumn{4}{c}{ Ingrediente } \\
\cline { 2 - 7 } Nutriente (\%) & $\begin{array}{c}\text { Alimento } \\
\text { balanceado }\end{array}$ & $\begin{array}{c}\text { Pulpa cítrica } \\
\text { deshidratada }\end{array}$ & $\begin{array}{c}\text { Harina de } \\
\text { soya }\end{array}$ & Melaza & Pasto estrella & $\begin{array}{c}\text { Ensilaje de } \\
\text { maíz }\end{array}$ \\
\hline MS (mín.) & 87,0 & 86,5 & 88,0 & 75,9 & 25,0 & 30,5 \\
PC (mín.) & 16,0 & 4,0 & 47,7 & 3,0 & 13,9 & 12,2 \\
EE (mín.) & 3,00 & 1,0 & 1,0 & 0,2 & --- & --- \\
Cenizas & --- & --- & --- & 11,4 & 11,4 & 12,4 \\
FDN & 12,0 & 14,0 & 9,8 & 0,1 & 63,9 & 52,2 \\
FDA & --- & --- & 6,2 & 0,1 & 36,0 & 35,8 \\
EN ${ }_{\mathrm{L}}$ Mcal kg-1 (mín.) & 1,8 & 1,8 & 2,2 & 1,8 & 1,4 & 1,5 \\
\hline
\end{tabular}

MS: materia seca, PC: proteína cruda, EE: extracto etéreo, FDN: fibra en detergente neutro, FDA: fibra en detergente ácida, EN ${ }_{\mathrm{L}}$ : energía neta de lactancia. / MS: dry matter, PC: crude protein, EE: ether extract, FDN: neutral detergent fiber, FDA: acid detergent fiber, $\mathrm{EN}_{\mathrm{L}}$ : net energy of lactation.

La composición química de las dietas ofrecidas para el grupo control y el tratamiento se presentan en el Cuadro 2.

\section{Tratamientos}

Se utilizó un diseño experimental alternativo o "cross-over", conocido como diseño 2x2 (dos tratamientos, dos periodos). Un grupo de quince animales recibieron el tratamiento A y posteriormente, al cambiar de periodo recibieron el tratamiento B; mientras que el otro grupo, también de quince animales, recibió los tratamientos en orden inverso. Entre los dos periodos, hubo un tiempo de adaptación y eliminación de efectos residuales de veintiún días.

\footnotetext{
$4 \quad$ Método que consiste en estimar el consumo diario por animal de materia seca del forraje, a partir de los datos de producción animal, los que a su vez determinan las necesidades de energía de los animales.
} 
Cuadro 2. Composición química de las dietas utilizadas durante el ensayo sobre el efecto que tiene el uso de un complejo de vitaminas B sobre la producción láctea y el metabolismo de vacas lecheras raza Jersey, en pastoreo. Cartago, Costa Rica. 2017.

Table 2. Chemical composition of the diets used during the test on the effect that the use of a vitamin B complex has on milk production and the metabolism of grazing Jersey dairy cattle. Cartago, Costa Rica. 2017.

\begin{tabular}{|c|c|c|}
\hline & \multicolumn{2}{|c|}{ Dieta } \\
\hline & Tratamiento & Control \\
\hline \multicolumn{3}{|l|}{ Ingrediente (\% de la MS) } \\
\hline Pasto estrella africana & 32,43 & 32,43 \\
\hline Ensilaje de maíz & 33,11 & 33,11 \\
\hline Alimento balanceado & 26,67 & 26,67 \\
\hline Harina de soya & 3,00 & 3,00 \\
\hline Pulpa cítrica deshidratada & 2,96 & 2,96 \\
\hline Melaza de caña & 1,81 & 1,81 \\
\hline \multirow[t]{2}{*}{ Suplemento de vitaminas } & 0,034 & --- \\
\hline & \multicolumn{2}{|c|}{ g $100 \mathrm{~g}^{-1}$ de MS } \\
\hline \multicolumn{3}{|l|}{ Composición química } \\
\hline MS & 33,13 & 33,13 \\
\hline $\mathrm{PC}$ & 13,34 & 13,34 \\
\hline FDN & 53,85 & 53,85 \\
\hline FDA & 32,65 & 32,65 \\
\hline $\mathrm{EN}_{\mathrm{L}}$ & 20,86 & 20,86 \\
\hline \multicolumn{3}{|l|}{ Consumo } \\
\hline MS, kg día ${ }^{-1}$ & 14,58 & 14,58 \\
\hline
\end{tabular}

MS: materia seca, PC: proteína cruda, FDN: fibra en detergente neutro, FDA: fibra en detergente acida, $\mathrm{EN}_{\mathrm{L}}$ : energía neta de lactancia. / MS: dry matter, PC: crude protein, FDN: neutral detergent fiber, FDA: acid detergent fiber, $\mathrm{EN}_{\mathrm{L}}$ : net energy of lactation.

El tratamiento A consistió en la inclusión de un complejo de vitaminas del complejo B sobrepasante del rumen, suministrado a una dosis de 3,0 g por vaca por día, mezclado con $10 \mathrm{~g}$ de alimento como vehículo. El suplemento de vitaminas del complejo B utilizado en la prueba fue una mezcla comercial que contenía 3,2 mg de biotina; 1,0 mg de ácido fólico; $10 \mathrm{mg}$ de ácido pantoténico; $25 \mathrm{mg}$ de piridoxina y 3,0 $\mathrm{mg}$ de cianocobalamina por gramo de producto, por lo que los animales con tratamiento recibieron diariamente una dosis total de 9,6 $\mathrm{mg}$ de biotina, 3,0 $\mathrm{mg}$ de ácido fólico, $30 \mathrm{mg}$ de ácido pantoténico, $75 \mathrm{mg}$ de piridoxina y $9 \mathrm{mg}$ de cianocobalamina. El tratamiento $\mathrm{B}$ o control consistió en suministrar a los animales únicamente $10 \mathrm{~g}$ de alimento balanceado como placebo.

El experimento tuvo una duración de once semanas, dividido en dos periodos de un mes cada uno. Durante el periodo 1, los animales del grupo 1 recibieron el tratamiento A y los animales del grupo 2 recibieron el tratamiento B. En el periodo 2 se intercambiaron los tratamientos.

\section{Recolección de muestras y resultados}

La producción de leche de todas las vacas se registró diariamente (am y pm) con un sistema electrónico que recopilaba la información en una base de datos. Se tomaron muestras individuales de leche por vaca durante el ordeño de la mañana y de la tarde, las cuales se refrigeraban y luego se enviaron al Laboratorio de Calidad de Leche de la Cooperativa de Productores de Leche Dos Pinos para su análisis. A las muestras se le aplicaron análisis de composición de la leche (porcentaje de sólidos totales, grasa, proteína y lactosa), conteo de células somáticas y concentración de nitrógeno ureico en leche (NUL). 
Para la determinación de los componentes de la leche, se empleó la metodología descrita por la FOSS Electric A/S para el equipo MilkoScanTM FT 120 Type 71200, en donde se utilizó $8 \mathrm{ml}$ de leche para la determinación automática de las variables bromatológicas. Los conteos de células somáticas (CS) se realizaron según la metodología descrita para el equipo Fossomatic Serie 400, en donde, $10 \mathrm{ml}$ de leche con la adición automática del colorante buferizado básico (Bromuro de etidio $(0,05 \%$ p/v) y Tritón X-100 concentrado $(1 \%$ p/v)), se calentaron por 15 min entre 37 a $42{ }^{\circ} \mathrm{C}$ y se analizaron de forma automática por el equipo. Este contabiliza los impulsos eléctricos que produce cada célula por medio de una reacción de fluorescencia del complejo célula-colorante, posterior a la estimulación por medio de una lámpara. La concentración de NUL se determinó mediante espectrofotometría.

En los últimos dos días de cada periodo y después de administrar el tratamiento $(12: 00 \mathrm{md})$, se tomaron muestras de sangre de los animales. La toma se hizo de la vena coccígea mediante vacutainer y se depositó en tubos sin anticoagulante debidamente identificados con el número del animal. Inmediatamente después, en el sitio, se realizó a cada muestra las pruebas de glucosa y beta-hidroxibutirato (BHBA). La medición de la concentración de glucosa y BHBA se realizó con un medidor electroquímico de mano, mediante la inmersión del dispositivo sensible desechable del equipo dentro del tubo de ensayo que contenía la sangre objeto de análisis. Todas las muestras se guardaron en refrigeración para su traslado al laboratorio clínico donde se midieron las concentraciones de aspartato amino transferasa (AST), nitrógeno ureico y albúmina.

\section{Análisis estadísticos}

Las vacas se mantuvieron con la misma dieta durante todo el tiempo de prueba, con el fin de evitar la variabilidad y que el suplemento de vitaminas del complejo B sobrepasante al rumen fuera la única diferencia.

Todos los resultados obtenidos se digitaron en una plantilla de Excel con las variables requeridas para su posterior análisis en el programa estadístico SAS. Los análisis se llevaron a cabo mediante un modelo mixto (PROC MIXED) con el animal como variable aleatoria. El modelo utilizado fue el siguiente:

$Y_{i j l m}=P_{i}+T_{j}+D_{l}+O_{m}+v_{i j l m}+e_{i j l m}$

En donde:

$\mathrm{Y}_{\mathrm{ijlm}}=$ es la variable de respuesta que se desea medir.

$\mathrm{P}_{\mathrm{i}}=$ es el periodo ( 1 o 2 ).

$\mathrm{T}_{\mathrm{j}}=$ tratamiento aplicado (con o sin suplementación de vitaminas B).

$\mathrm{D}_{1}=$ día de medición.

$\mathrm{O}_{\mathrm{m}}=$ ordeño $(\mathrm{AM}$ o PM).

$\mathrm{v}_{\mathrm{ijlm}}=$ vaca.

$\mathrm{e}_{\mathrm{ijlm}}=$ error estándar.

\section{Resultados}

\section{Producción y composición láctea}

No se encontraron diferencias significativas $(p>0,05)$ entre tratamientos para la producción diaria de leche (Cuadro 3). En el periodo 1 el grupo con tratamiento (vitaminas B) produjo en promedio 15,8 $\pm 1,0$ litros por vaca por día, con producciones que variaron entre 15,1 a 16,7 l de leche. Durante el mismo periodo, el grupo control produjo un promedio diario de $16,1 \pm 1,01$, valores que variaron entre 15,7 y 16,6 . 
Cuadro 3. Promedios de producción y composición láctea de cada periodo por ordeño y por día de vacas lecheras raza Jersey en pastoreo, para los tratamientos de suplementación del complejo de vitaminas B. Cartago, Costa Rica. 2017.

Table 3. Average milk production and milk composition per milking and per day of grazing Jersey dairy cows, for the supplementation treatments of a vitamin B complex. Cartago, Costa Rica. 2017.

\begin{tabular}{|c|c|c|c|c|c|c|c|c|c|}
\hline Periodo & Ordeño & Tratamiento & $\begin{array}{l}\text { Producción } \\
\text { (l) }\end{array}$ & $\begin{array}{c}\text { Sólidos } \\
\text { totales (\%) }\end{array}$ & $\begin{array}{c}\text { Grasa } \\
(\%)\end{array}$ & $\begin{array}{c}\text { Proteína } \\
(\%)\end{array}$ & $\begin{array}{c}\text { Lactosa } \\
(\%)\end{array}$ & $\begin{array}{c}\text { CCS } \\
(\log 10)\end{array}$ & $\begin{array}{c}\text { NUL } \\
\left(\mathbf{m g ~ d l}^{-1}\right)\end{array}$ \\
\hline \multirow{6}{*}{1} & \multirow{2}{*}{$\mathrm{AM}$} & Vitaminas B & $11,2 \pm 0,6$ & $12,8 \pm 0,3$ & $4,2 \pm 0,4$ & $3,5 \pm 0,1$ & $4,5 \pm 0,1$ & $5,1 \pm 0,2$ & $16,4 \pm 0,6$ \\
\hline & & Control & $11,4 \pm 0,6$ & $12,7 \pm 0,3$ & $4,1 \pm 0,4$ & $3,6 \pm 0,1$ & $4,5 \pm 0,1$ & $5,2 \pm 0,1$ & $15,6 \pm 0,6$ \\
\hline & \multirow{2}{*}{ PM } & Vitaminas B & $4,80 \pm 0,6$ & $14,6 \pm 0,3$ & $6,2 \pm 0,4$ & $3,5 \pm 0,1$ & $4,4 \pm 0,1$ & $5,4 \pm 0,2$ & $17,5 \pm 0,6$ \\
\hline & & Control & $4,90 \pm 0,6$ & $14,4 \pm 0,3$ & $5,8 \pm 0,4$ & $3,5 \pm 0,1$ & $4,4 \pm 0,1$ & $5,6 \pm 0,1$ & $16,6 \pm 0,6$ \\
\hline & \multirow{2}{*}{ Día } & Vitaminas B & $16,0 \pm 0,6$ & $13,7 \pm 0,3$ & $5,2 \pm 0,4$ & $3,5 \pm 0,1$ & $4,4 \pm 0,1$ & $5,2 \pm 0,2$ & $16,9 \pm 0,6$ \\
\hline & & Control & $16,2 \pm 0,6$ & $13,5 \pm 0,3$ & $4,9 \pm 0,4$ & $3,5 \pm 0,1$ & $4,4 \pm 0,1$ & $5,4 \pm 0,1$ & $16,1 \pm 0,6$ \\
\hline \multirow{6}{*}{2} & \multirow{2}{*}{$\mathrm{AM}$} & Vitaminas B & $9,80 \pm 0,6$ & $12,5 \pm 0,4$ & $3,8 \pm 0,4$ & $3,8 \pm 0,1$ & $4,4 \pm 0,1$ & $5,4 \pm 0,1$ & $18,2 \pm 0,6$ \\
\hline & & Control & $9,60 \pm 0,6$ & $13,0 \pm 0,4$ & $4,2 \pm 0,4$ & $3,7 \pm 0,1$ & $4,5 \pm 0,1$ & $5,2 \pm 0,2$ & $18,3 \pm 0,6$ \\
\hline & \multirow{2}{*}{ PM } & Vitaminas B & $4,20 \pm 0,6$ & $14,8 \pm 0,4$ & $6,1 \pm 0,4$ & $3,7 \pm 0,1$ & $4,4 \pm 0,1$ & $5,5 \pm 0,1$ & $16,3 \pm 0,6$ \\
\hline & & Control & $4,20 \pm 0,6$ & $15,5 \pm 0,4$ & $6,8 \pm 0,4$ & $3,6 \pm 0,1$ & $4,4 \pm 0,1$ & $5,4 \pm 0,2$ & $16,6 \pm 0,6$ \\
\hline & \multirow{2}{*}{ Día } & Vitaminas B & $14,1 \pm 0,6$ & $13,7 \pm 0,4$ & $5,0 \pm 0,4$ & $3,8 \pm 0,1$ & $4,4 \pm 0,1$ & $5,4 \pm 0,1$ & $17,3 \pm 0,6$ \\
\hline & & Control & $13,7 \pm 0,6$ & $14,3 \pm 0,4$ & $5,5 \pm 0,4$ & $3,7 \pm 0,1$ & $4,5 \pm 0,1$ & $5,3 \pm 0,2$ & $17,5 \pm 0,6$ \\
\hline
\end{tabular}

CCS: conteo de células somáticas, NUL: Nitrógeno ureico en leche / CCS: somatic cell count, NUL: milk urea nitrogen.

En el periodo 2, el grupo que consumió suplemento vitamínico obtuvo una producción diaria promedio de $14,1 \pm 1,01$ con un rango que varió de 13,6 a 14,8 1, mientras que el grupo control obtuvo un promedio de $13,7 \pm 1,0$ 1 por día y mostró un rango desde 13,1 hasta 14,6 1.

Con respecto a la composición de la leche, no se encontraron diferencias significativas $(p>0,05)$ entre tratamientos para las diferentes variables evaluadas (Cuadro 3). Al finalizar el periodo 1, el grupo de vacas suplementadas con vitaminas tuvo un porcentaje de sólidos totales de $13,7 \pm 0,3,5,2 \pm 0,4 \%$ de grasa, $3,5 \pm 0,1 \%$ de

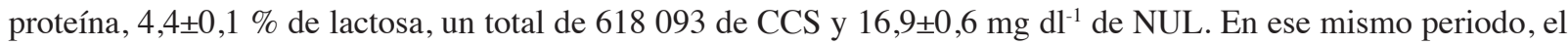
grupo control tuvo $13,5 \pm 0,3 \%$ de sólidos totales, $4,9 \pm 0,4 \%$ de grasa, $3,5 \pm 0,1 \%$ de proteína, $4,4 \pm 0,1 \%$ de lactosa, 558325 de CCS y 16,1 $\pm 0,1 \mathrm{mg} \mathrm{dl}^{-1}$ de NUL. Para el periodo 2 , los resultados para el grupo con suplemento de vitaminas fue de un $13,7 \pm 0,4 \%$ de sólidos totales, $5,0 \pm 0,4 \%$ de grasa, $3,8 \pm 0,1 \%$ de proteína, $4,4 \pm 0,1 \%$ de lactosa,

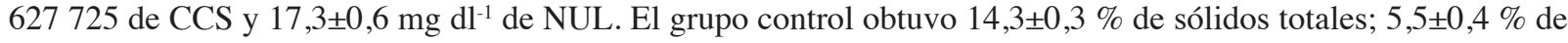
grasa, $3,7 \pm 0,1 \%$ de proteína, $4,5 \pm 0,1 \%$ de lactosa, 411496 de CCS y 17,5 $\pm 0,6 \mathrm{mg} \mathrm{dl}^{-1}$ de NUL.

\section{Parámetros en sangre}

Los resultados de los parámetros de la química sanguínea se presentan en el Cuadro 4. No se encontraron diferencias significativas $(\mathrm{p}>0,05)$ entre tratamientos en ningún periodo para las diferentes variables evaluadas. Al

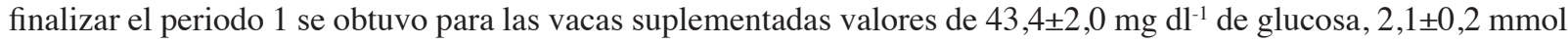

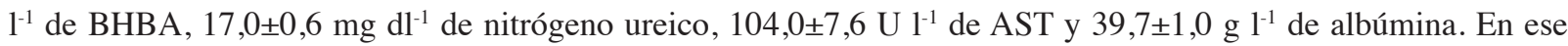

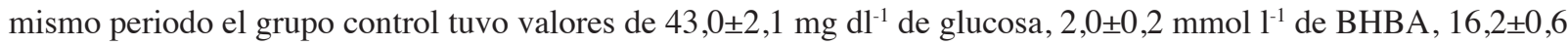
mg dl ${ }^{-1}$ de nitrógeno ureico, 106,6 $\pm 7,8 \mathrm{U} \mathrm{l}^{-1}$ de AST y $38,1 \pm 1,0 \mathrm{~g} \mathrm{l}^{-1}$ de albúmina.

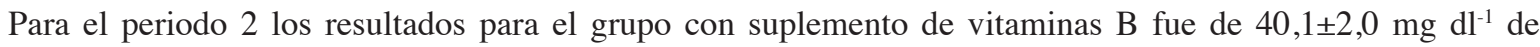

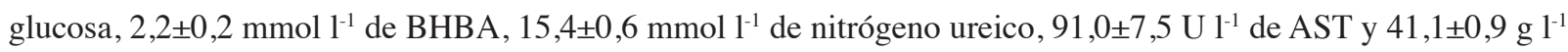
de albúmina. El grupo control presentó concentraciones de glucosa de 41,4 $\pm 2,1 \mathrm{mg} \mathrm{dl}^{-1}, 2,2 \pm 0,2 \mathrm{mmol} \mathrm{l}^{-1} \mathrm{de}$ BHBA,

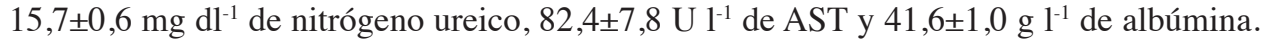


Cuadro 4. Valores promedio y error estándar de la química sanguínea, de los últimos dos días de cada periodo y su promedio, para vacas lecheras raza Jersey, en pastoreo, con tratamiento de suplementación del complejo de vitaminas B. Cartago, Costa Rica. 2017. Table 4. Average values and standard error of blood chemistry, of the last two days of each period and its average, for grazing Jersey dairy cows with the supplementation treatment of a vitamin B complex. Cartago, Costa Rica. 2017.

\begin{tabular}{|c|c|c|c|c|c|c|c|}
\hline Periodo & Día & Tratamiento & $\begin{array}{r}\text { Glucosa } \\
\left(\mathbf{m g ~ d l}^{-1}\right)\end{array}$ & $\begin{array}{c}\text { BHB } \\
\left(\mathrm{mmol} \mathrm{l}^{-1}\right)\end{array}$ & $\begin{array}{l}\text { N ureico } \\
\left(\mathrm{mg} \mathrm{dl}^{-1}\right)\end{array}$ & $\begin{array}{c}\text { AST } \\
\left(\mathbf{U ~}^{\left.\mathbf{l}^{-1}\right)}\right.\end{array}$ & $\begin{array}{l}\text { Albúmina } \\
\qquad\left(\mathrm{g} \mathrm{l}^{-1}\right)\end{array}$ \\
\hline \multirow{6}{*}{1} & \multirow{2}{*}{1} & Vitaminas B & $40,2 \pm 2,1$ & $2,5 \pm 0,2$ & $16,5 \pm 0,6$ & $96,2 \pm 7,8$ & $39,9 \pm 1,0$ \\
\hline & & Control & $41,2 \pm 2,0$ & $2,4 \pm 0,2$ & $16,0 \pm 0,6$ & $99,9 \pm 7,5$ & $37,8 \pm 0,9$ \\
\hline & \multirow{2}{*}{2} & Vitaminas B & $47,4 \pm 2,1$ & $1,7 \pm 0,2$ & $18,0 \pm 0,6$ & $112,3 \pm 7,8$ & $39,7 \pm 1,0$ \\
\hline & & Control & $44,2 \pm 2,0$ & $1,7 \pm 0,2$ & $16,0 \pm 0,6$ & $112,6 \pm 7,5$ & $38,3 \pm 0,9$ \\
\hline & \multirow{2}{*}{ Promedio } & Vitaminas B & $43,4 \pm 2,0$ & $2,1 \pm 0,2$ & $17,0 \pm 0,6$ & $104,0 \pm 7,6$ & $39,7 \pm 1,0$ \\
\hline & & Control & $43,0 \pm 2,1$ & $2,0 \pm 0,2$ & $16,2 \pm 0,6$ & $106,6 \pm 7,8$ & $38,1 \pm 1,0$ \\
\hline \multirow{6}{*}{2} & \multirow{2}{*}{1} & Vitaminas B & $39,2 \pm 2,0$ & $2,2 \pm 0,2$ & $14,0 \pm 0,6$ & $89,3 \pm 7,5$ & $37,4 \pm 0,9$ \\
\hline & & Control & $40,6 \pm 2,1$ & $2,3 \pm 0,2$ & $14,2 \pm 0,6$ & $86,6 \pm 7,8$ & $37,7 \pm 1,0$ \\
\hline & \multirow{2}{*}{2} & Vitaminas B & $41,0 \pm 2,2$ & $2,2 \pm 0,2$ & $16,5 \pm 0,6$ & $92,6 \pm 7,5$ & $44,8 \pm 0,9$ \\
\hline & & Control & $42,2 \pm 2,1$ & $2,1 \pm 0,2$ & $16,8 \pm 0,6$ & $78,3 \pm 7,8$ & $45,5 \pm 1,0$ \\
\hline & \multirow{2}{*}{ Promedio } & Vitaminas B & $40,1 \pm 2,0$ & $2,2 \pm 0,2$ & $15,4 \pm 0,6$ & $91,0 \pm 7,5$ & $41,1 \pm 0,9$ \\
\hline & & Control & $41,4 \pm 2,1$ & $2,2 \pm 0,2$ & $15,7 \pm 0,6$ & $82,4 \pm 7,8$ & $41,6 \pm 1,0$ \\
\hline
\end{tabular}

BHB: Beta-hidroxibutirato, N ureico: Nitrógeno ureico, AST: Aspartato aminotransferasa / BHB: Beta-hidroxibutirate, $\mathrm{N}$ ureico: urea nitrogen, AST: aspartate aminotransferase.

\section{Discusión}

El no haber encontrado una mejoría en la producción de leche concuerda con los resultados obtenidos por Ferreira et al. (2007), quien al suministrar $20 \mathrm{mg}$ de biotina al día no observó aumentos en la producción o componentes lácteos en vacas con producciones de leche de $24 \mathrm{~kg}_{\text {día }}{ }^{-1}$.

La biotina es la vitamina que más se ha estudiado por su efecto benéfico en la salud de la pezuña (Mülling et al., 1999) y diversas investigaciones han demostrado que el suplementar $20 \mathrm{mg}$ de esta vitamina por día aumentó la producción de leche (Chen et al., 2011; Enjalbert et al., 2008; Lean \& Rabiee, 2011), lo cual difiere con los resultados obtenidos en la presente investigación, situación que pudo deberse a que la dosis utilizada en el presente estudio fue la mitad (9,6 mg día $\left.{ }^{-1}\right)$ de la dosis comúnmente usada (20 mg día $\left.{ }^{-1}\right)$; ya que se considera que, al estar recubierta, va a llegar al intestino delgado en su totalidad y a que la mayoría de estudios consultados se llevaron a cabo en vacas altamente productoras de la raza Holstein y en sistemas de estabulación completo.

También, el periodo de suplementación utilizado en el presente experimento fue de cuatro semanas en cada periodo y se ha visto que se requieren periodos más extensos de suplementación con biotina para observar un efecto benéfico en la salud de la pezuña. Al respecto, se han observado reducciones en las lesiones de la pezuña después de 2 o 3 meses de suplementación con biotina (Hoblet et al., 2002), pero un estudio mostró que se requieren seis meses de suplementación para ver una reducción significativa en las lesiones de línea blanca en el casco (Pötzsch et al., 2003). La explicación es porque la renovación del tejido del casco en las vacas tarda alrededor de 3 a 4 meses (Fitzgerald et al., 2000).

En cuanto a no haber encontrado diferencias significativas con respecto al porcentaje y producción de grasa láctea es concordante con los resultados de un primer experimento de Sacadura et al. (2008), donde el suplementar 
una mezcla protegida de vitaminas B a vacas en lactación media, no generó ninguna respuesta en el porcentaje de grasa láctea e inclusive este fue más bajo que el del grupo control (3,63 vs 3,71 \%).

Los resultados obtenidos de la presente investigación no concuerdan con un segundo experimento de Saccadura et al. (2008), donde el suplementar 3,0 g de vitaminas B protegidas aumentó la producción de grasa láctea en vacas en lactación temprana (1,47 vs $\left.1,40 \mathrm{~kg} \mathrm{día}^{-1}\right)$.

En cuanto a la concentración de proteína láctea, los valores fueron similares para ambos grupos $(\mathrm{p}>0,05)$ durante la fase experimental, situación que coincide con un estudio realizado en vacas multíparas en etapa de lactación temprana (Juchem et al., 2012), donde no se encontraron diferencias en cuanto a la producción de proteína láctea al suplementar un complejo de vitaminas B recubierto con la misma composición que el utilizado en el presente experimento.

Los porcentajes de lactosa estuvieron dentro del rango de 4,40 y 4,50\% para los dos grupos a lo largo de los dos periodos experimentales, situación que ya se ha reportado (Juchem et al., 2012), pero con un valor mayor (4,7 \%). Este valor superior de lactosa se puede deber al uso de diferentes razas, ya que vacas de la raza Holstein presentan producciones de leche mayores en comparación con vacas Jersey. Se ha visto que la tasa de secreción de agua está determinada por la tasa de síntesis de lactosa en la glándula mamaria, debido a que la lactosa es el principal factor responsable de la osmolaridad de la leche (Miglior et al., 2006). Por lo tanto, la síntesis de lactosa regula la secreción de agua y consecuentemente la producción de leche (Linn, 1988). Al estar involucrada directamente en la producción láctea, la lactosa es el componente lácteo menos variable y se mantiene en concentraciones alrededor de 4,6\%. Además, la lactosa no está sujeta a grandes cambios a través de la manipulación genética o nutricional (Linn, 1988).

El suplementar a las vacas con un complejo de vitaminas B recubierto en este experimento no afectó los

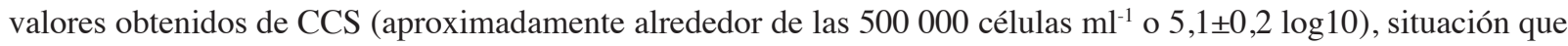
concuerda con el primer experimento de Saccadura et al. (2008), en donde no hubo diferencia en el CCS entre el control y el tratamiento de 3,0 g de vitaminas B protegidas (281000 CCS).

Un CCS > 200000 células $\mathrm{ml}^{-1}$ es indicativo de que las vacas tienen una infección intramamaria, un conteo $<200000$ células $\mathrm{ml}^{-1}$ muestra que el animal está manejando adecuadamente la exposición a patógenos y un CCS

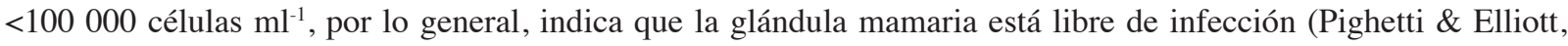
2011). Los animales en el presente experimento presentaron valores superiores a 200000 células $\mathrm{ml}^{-1}$, por lo que se clasificarían con infección mamaria. Se ha visto que concentraciones altas de ácidos grasos no esterificados (AGNE) y betahidroxibutirato (BHBA) se han asociado a una función inmune deteriorada y mastitis en vacas lecheras (Burvenich et al., 2007). Las vacas del experimento mostraron concentraciones altas de BHBA, por lo que podían haber estado sufriendo de una función inmune alterada y esto predisponía a una colonización bacteriana excesiva que se refleja en el elevado CCS.

Los valores obtenidos para glucosa y BHBA no mostraron diferencias significativas ( $>>0,05$ ), lo que concuerda con lo concluido por Richards et al. (2002), que al utilizar un complejo de varias vitaminas B (que tenía vitamina B12, biotina, ácido fólico) no afectó los valores de AGNE, BHBA, triglicéridos o glucosa en el plasma sanguíneo de los animales. Al suplementar biotina sola o con un complejo de vitaminas B se encontró que no se dieron diferencias en los valores plasmáticos de glucosa, AGNE y BHBA en comparación con el grupo control (Majee et al., 2003).

En cuanto al ácido fólico se puede ver que su suplementación afectó positivamente las concentraciones de glucosa en sangre. Las vacas suplementadas con ácido fólico protegido $(0,1,2$ y 3 g por día) incrementaron linealmente las concentraciones en el plasma de glucosa, mientras que las concentraciones de AGNE y BHBA disminuyeron linealmente ( $\mathrm{Li}$ et al., 2016). Durante el posparto las concentraciones de glucosa e insulina fueron mayores y las concentraciones de AGNE fueron menores en vacas que recibieron un suplemento semanal de ácido fólico en comparación con las que no recibieron ninguna suplementación (Duplessis et al., 2017). 
El objetivo del experimento a la hora de medir glucosa y BHBA en sangre era valorar el balance en el metabolismo energético de las vacas. En el presente experimento, los valores de glucosa en sangre estuvieron en promedio entre 39,2 y 47,4 $\mathrm{mg} / \mathrm{dl}$ (mediciones posprandiales). Los valores de referencia para las mediciones de

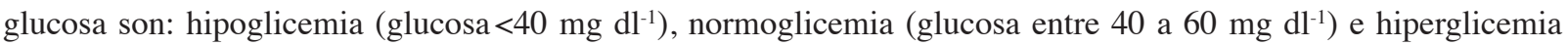
(glucosa $>60 \mathrm{mg} \mathrm{dl}^{-1}$ ) (Mair et al., 2016), por lo que los animales utilizados en el ensayo estuvieron en el límite inferior de la normoglicemia o en hipoglicemia.

Los animales presentaron valores de BHBA de 1,7 a 2,5 $\mathrm{mmol} \mathrm{l}^{-1}$. El valor de 1,2 $\mathrm{mmol} \mathrm{l}^{-1}$ se considera el límite para diferenciar entre un animal saludable y otro que presente cetosis subclínica (Enjalbert et al., 2001). Por lo que se observa que las vacas se encontraban en un grado de cetosis subclínica, lo cual se ve respaldado por las bajas concentraciones de glucosa posprandiales.

Un estudio realizado por Bjerre-Harpøth et al. (2012) concluyó que las concentraciones de BHBA en plasma estaban negativamente relacionadas con las concentraciones de glucosa plasmáticas. Otra investigación demostró que las concentraciones de BHBA disminuyeron después de administrar un bolo de glucosa intravenoso (500 mg $\mathrm{kg}^{-1}$ ) a las vacas en lactación temprana, media y cerca del secado y que la producción de cuerpos cetónicos se redujo (Chalmeh et al., 2015).

En este experimento el suplementar 3,0 g de un complejo de vitaminas B sobrepasante no mostró diferencias significativas en los valores de nitrógeno ureico (en plasma y leche) de los animales en comparación con el grupo control. Esto coincide con los resultados de una investigación en la que al suplementar biotina sola o con un complejo de vitaminas B, encontraron que no se dieron diferencias significativas en las concentraciones de nitrógeno ureico en leche (NUL) (valores entre 12,2 y 13,2 $\mathrm{mg} \mathrm{dl}^{-1}$ ) en comparación con el grupo control (Majee et al., 2003). Asimismo, al utilizar un complejo de varias vitaminas B, no se encontraron diferencias significativas en los valores de urea en plasma sanguíneo de los animales suplementados con respecto al control (Richards et al., 2002). Solamente en un experimento donde se suplementó diariamente 0 , 2, y 4,0 $\mathrm{mg}$ de ácido fólico por kilogramo de peso corporal, se observó que las vacas multíparas mostraron una disminución en el NUL en comparación con vacas no suplementadas, lo cual significó una utilización de nitrógeno más eficiente que las vacas sin suplementación (Girard \& Matte, 1996).

Los resultados de nitrógeno ureico obtenidos en el presente experimento estaban dentro del rango de los 15,6 a $18,3 \mathrm{mg} \mathrm{dl}^{-1}$ en leche y de 14,0 a $18,0 \mathrm{mg} \mathrm{dl}^{-1}$ en sangre, concentraciones indicativas de una insuficiente suplementación energética en la dieta de los animales. En este caso específico, los animales requerían aproximadamente de 23-24 Mcal kg ${ }^{-1}$ MS y la dieta contenía en promedio 20,86 $\mathrm{Mcal} \mathrm{kg}^{-1} \mathrm{MS}$.

Al evaluar la AST y la albúmina se mide si el complejo de vitaminas B tiene alguna influencia sobre el funcionamiento del hígado y sus enzimas, sobre lo cual se ha reportado una baja actividad de una sola o de dos, carboxilasas gluconeogénicas en el hígado de ratas cuando existe una deficiencia de biotina (Mock \& Mock, 2002; Rodríguez et al., 2001). Los valores obtenidos de AST (78,3-112,6 U l-1) y de albúmina (37,4 - 45,5 $\left.\mathrm{g} \mathrm{l}^{-1}\right)$ no variaron con la suplementación del complejo de vitaminas B recubierto. Los valores de referencia en bovinos para la AST son de 78 - $132 \mathrm{U} \mathrm{l}^{-1}$ y de albúmina de 21 - $36 \mathrm{~g} \mathrm{l}^{-1}$ (Radostits et al., 2000). Por lo que se puede observar, que los resultados obtenidos se encuentran dentro de los valores normales para bovinos y la suplementación de vitaminas no tuvo influencia sobre la actividad de las enzimas hepáticas y su funcionamiento. Esto concuerda nuevamente con que no se haya visto diferencia en las concentraciones de glucosa, ya que estas enzimas se encuentran en el hígado y participan en el metabolismo gluconeogénico.

\section{Conclusión}

El suplementar un complejo de vitaminas B recubierto a dosis diarias de 9,6 mg de biotina, 3,0 mg de ácido fólico, $30 \mathrm{mg}$ de ácido pantoténico, $75 \mathrm{mg}$ de piridoxina y $9 \mathrm{mg}$ de cianocobalamina, no generó ninguna respuesta medible en los parámetros productivos o sanguíneos de los animales suplementados con respecto al grupo control. 


\section{Agradecimiento}

Los autores agradecen el financiamiento parcial ofrecido por las empresas JEFO y FARYVET.

\section{Referencias}

Baker, R. D. (1982). Estimation of herbage intake from animal performance. In: J.D. Leaver (Ed.), Herbage intake handbook (pp. 77-93). The British Grassland Society.

Baron, R. B. (2016). Nutritional disorders. In M. A. Papadakis, S. J. McPhee, \& M. W. Rabow (Eds.), Current medical diagnosis and treatment (pp. 1249-1266). McGraw-Hill.

Bender, D. A. (2015). Micronutrients: Vitamins and minerals. In V. W. Rodwell, D. A. Bender, K. M. Botham, P. J. Kennelly, \& P. A. Weil (Eds.), Harper's Illustrated Biochemistry (pp. 546-563). 30th ed. McGraw-Hill.

Bergman, E. N., Brockman, R. P., \& Kaufman, C. F. (1974). Glucose metabolism in ruminants: comparison of whole-body turnover with production by the gut, liver and kidneys. Federation Proceedings, 33(7), 1849-1854.

Bjerre-Harpøth, V., Friggens, N. C., Thorup, V. M., Larsen, T., Damgaard, B. M., Ingvartsen, K. L., \& Moyes, K. M. (2012). Metabolic and production profiles of dairy cows in response to decreased nutrient density to increase physiological imbalance at different stages of lactation. Journal of Dairy Science, 95(5), 2362-2380. http://doi.org/10.3168/ jds.2011-4419

Burvenich, C., Bannerman, D. D., Lippolis, J.D., Peelman, L., Nonnecke, B. J., Kehrli, M.E., \& Paape, M. J. (2007). Cumulative physiological events influence the inflammatory response of the bovine udder to Escherichia coli infections during the transition period. Journal of Dairy Science, 90(E Suppl.), E39-E54. http://doi.org/10.3168/jds.2006-696

Cassell, B. G. (2001). Optimal genetic improvement for the high producing cow. Journal of Dairy Science, 84(E. Suppl.), E144-E150. https://doi.org/10.3168/jds.S0022-0302(01)70208-1

Chalmeh, A., Pourjafar, M., \& Nazifi, S. (2015). Evaluating the metabolism of high producing Holstein dairy cows following bolus intravenous glucose administration. Veterinarski Arhiv, 85(6), 621-633.

Chen, B., Wang, C., Wang, Y. M., \& Liu, J. X. (2011). Effect of biotin on milk performance of dairy cattle: A meta-analysis. Journal of Dairy Science, 94(7), 3537-3546. http://doi.org/10.3168/jds.2010-3764

Duplessis, M., Lapierre, H., Pellerin, D., Laforest, J. P., \& Girard, C. L. (2017). Effects of intramuscular injections of folic acid, vitamin B, or both, on lactational performance and energy status of multiparous dairy cows. Journal of Dairy Science, 100(5), 4051-4064. https://doi.org/10.3168/jds.2016-12381

Elizondo, J. A., \& Boschini, C. (2001). Efecto de la densidad de siembra sobre el rendimiento y calidad del forraje de maíz. Agronomía Mesoamerica, 12(2), 181-187. http://doi.org/10.15517/am.v12i2.17231

Enjalbert, F., Nicot, M. C., Bayourthe, C., \& Moncoulon, R. (2001). Ketone bodies in milk and blood of dairy cows: Relationship between concentrations and utilization for detection of subclinical ketosis. Journal of Dairy Science, 84(3), 583-589. http://doi.org/10.3168/jds.S0022-0302(01)74511-0

Enjalbert, F., Nicot, M. C., \& Packington, A. J. (2008). Effects of peripartum biotin supplementation of dairy cows on milk production and milk composition with emphasis on fatty acids profile. Livestock Science, 114(2-3), 287-295. http:// doi.org/10.1016/j.livsci.2007.05.013 
Ferreira, G., Brown, A. N., \& Teets, C. L. (2015). Effect of biotin and pantothenic acid on performance and concentrations of avidin-binding substances in blood and milk of lactating dairy cows. Journal of Dairy Science, 98(9), 6449-6454. http://doi.org/10.3168/jds.2015-9620

Ferreira, G., Weiss, W. P., \& Willet, L. B. (2007). Changes in measures of biotin status do not reflect milk yield responses when dairy cows are fed supplemental biotin. Journal of Dairy Science, 90(3), 1452-1459. http://doi.org/10.3168/jds. S0022-0302(07)71630-2

Fitzgerald, T., Norton, B. W., Elliott, R., Podlich, H., \& Svendsen, O. L. (2000). The influence of long-term supplementation with biotin on the prevention of lameness in pasture fed dairy cows. Journal of Dairy Science, 83(2), 338-344. http:// doi.org/10.3168/jds.S0022-0302(00)74884-3

Girard, C. L., \& Matte, J. J. (1996). Effects of dietary supplements of folic acid on lactational performance of dairy cows. Journal of Dairy Science, 79(Suppl. 1), 199.

Hoblet, K., Weiss, W., Anderson, D., \& Moeschberger, M. (2002). Effect of oral biotin supplementation on hoof health in Holstein heifers during gestation and early lactation. In: J.K. Shearer (Ed.), XII International Symposium on Lameness in Ruminants (p. 253-255). Florida State University.

Juchem, S. O., Robinson, P., \& Evans, E. (2012). A fat based rumen protection technology post-ruminally delivers a B vitamin complex to impact performance of multiparous Holstein cows. Animal Feed Science and Technology, 174(1-2), 68-78. http://doi.org/10.1016/j.anifeedsci.2012.03.004

Lean, I. J., \& Rabiee, A. R. (2011). Effect of feeding biotin on milk production and hoof health in lactating dairy cows: A quantitative assessment. Journal of Dairy Science, 94(3), 1465-1476. http://doi.org/10.3168/jds.2010-3682

Leng, R. A. (1970). Glucose synthesis in ruminants. Advances in Veterinay Science and Comparative Medicine, 14, $209-260$.

Li, H. Q., Liu, Q., Wang, C., Yang, Z. M., Guo, G., Huo, W. J., Pei, C. X., Zhang, Y. L., Zhang, S. L., Wang, H., Liu, J. X., \& Huang, Y. X. (2016). Effects of dietary supplements of rumen-protected folic acid on lactation performance, energy balance, blood parameters and reproductive performance in dairy cows. Animal Feed Science and Technology, 213, 55-63. http://doi.org/10.1016/j.anifeedsci.2016.01.005

Linn, J. G. (1988). Factors affecting the composition of milk from dairy cows. In: National Research Council (Ed.), Designing foods: Animal product options in the marketplace (pp. 9-17). National Academy Press.

Lomax, M. A., \& Baird, G. D. (1983). Blood flow and nutrient exchange across the liver and gut of the dairy cow. British Journal of Nutrition, 49(3), 481-496. http://doi.org/10.1079/bjn19830057

Mair, B., Drillich, M., Klein-Jöbstl, D., Kanz, P., Borchardt, S., Meyer, L., \& Iwersen, M. (2016). Glucose concentration in capillary blood of dairy cows obtained by a minimally invasive lancet technique and determined with three different hand-held devices. BMC Veterinary Research, 12(34), 1-11. https://doi.org/10.1186/s12917-016-0662-3

Majee, D. N., Schwab, E. C., Bertics, S. J., Seymour, W. M., \& Shaver, R. D. (2003). Lactation performance by dairy cows fed supplemental biotin and a B-vitamin blend. Journal of Dairy Science, 86(6), 2106-2112. http://doi.org/10.3168/jds. S0022-0302(03)73800-4

McDowell, L. R. (2000). Vitamins in animal and human nutrition. Iowa State University Press and Wiley-Blackwell.

Miglior, F., Sewalem, A., Jamrozik, J., Lefebvre, D. M., \& Moore, R. K. (2006). Analysis of milk urea nitrogen and lactose and their effect on longevity in Canadian dairy cattle. Journal of Dairy Science, 89(12), 4886-4894. http://doi. org/10.3168/jds.S0022-0302(06)72537-1 
Mock D. M., \& Mock, N. I. (2002). Lymphocyte propionyl-CoA carboxylase is an early and sensitive indicator of biotin deficiency in rats, but urinary excretion of 3-hydroxy-propionic acid is not. Journal of Nutrition, 132(7), 1945-1950. http://doi.org/10.1093/jn/132.7.1945

Mülling, K. W., Bragulla, H. H., Reese, S., Budras, K. D., \& Steinberg, W. (1999). How structures in bovine hoof epidermis are influenced by nutritional factor. Anatomy, Histology, Embryology, 28(2), 103-108. http://doi.org/10.1046/j.14390264.1999.00180.x

NRC (National Research Council). (2001). Nutrient requirements of dairy cattle. 7th rev. ed. National Academy Press, WA.

Oltenacu, P., \& Broom, D. M. (2010). The impact of genetic selection for increased milk yield on the welfare of dairy cows. Animal Welfare, 19(1), 39-49.

Pighetti, G. M., \& Elliott, A. A. (2011). Gene polymorphisms: The keys for marker assisted selection and unraveling core regulatory pathways for mastitis resistance. Journal of Mammary Gland Biology and Neoplasia, 16, 421-432. http:// doi.org/10.1007/s10911-011-9238-9

Pötzsch, C. J., Collis, V. J., Blowey, R. W., Packington, A. J., \& Green, L. E. (2003). The impact on parity and duration of biotin supplementation on white line lameness in dairy cattle. Journal of Dairy Science, 86(8), 2577-2582. http://doi. org/10.3168/jds.S0022-0302(03)73852-1

Radostits, O. M., Gay, C. C., Blood, D. C., \& Hinchcliff, K. W. (2000). Veterinary Medicine. 9th ed. W.B. Saunders.

Richards, S. E., Hicklin, S., Lord, T., Nickson, A., Long, J., Brackenbury, J., \& Newbold, J. R. (2002). Effects of B vitamins and methyl group donors on milk production, milk composition and blood chemistry in dairy cows. British Society of Animal Sciences.

Richmonds, R. (2004). Vitamins. In S. Loue, \& M. Sajatovic (Eds.), Encyclopedia of women's health (pp. 679-682). Springer Science+Business Media.

Rodríguez, M. R., Cano, S., Mendez, S. T., \& Velazquez, A. (2001). Biotin regulates the genetic expression of holocarboxylase synthetase and mitochondrial carboxylases in rats. Journal of Nutrition, 131(7), 1909-1913. http://doi.org/10.1093/ jn/131.7.1909

Sacadura, F. C., Robinson, P. H., Evans, E., \& Loredo, M. (2008). Effects of a ruminally protected B-vitamin supplement on milk yield and composition of lactating dairy cows. Animal Feed Science and Technology, 144(1-2), 111-124. http:// doi.org/10.1016/j.anifeedsci.2007.10.005

Santschi, D. E., Berthiaume, R., Matte, J. J., Mustafa, A. F., \& Girard, C. L. (2005). Fate of supplementary B-vitamins in the gastrointestinal tract of dairy cows. Journal of Dairy Science, 88(6), 2043-2054. http://doi.org/10.3168/jds.S00220302(05)72881-2

Van-Knegsel, A. T. M, van-den-Brand, H., Dijkstra, H. J., Tamminga, S., \& Kemp, B. (2005). Effect of dietary energy source on energy balance, production, metabolic disorders and reproduction in lactating dairy cattle. Reproduction, Nutrition, Development, 45(6), 665-688. http://doi.org/10.1051/rnd:2005059 